\title{
EVALUATION OF TMJ OSTEOARTHRITIC OSSEOUS CHANGES USING DIFFERENT IMAGING MODALITIES: A DIAGNOSTIC ACCURACY STUDY
}

\author{
Iman Dakhli*
}

\begin{abstract}
Aim: This study was conducted to compare the diagnostic accuracy of CBCT imaging to digital panoramic imaging in assessment of osteoarthritic TMJ osseous changes.

Methods: 25 patients were enrolled in the current investigation, divided into two groups, Group (1) the diseased group including fifteen patients. Group (2) the control group including Ten normal asymptomatic healthy patients (20 joints) but they were seeking for treatment of other dental problems, in the two groups enrolled in this study all participants were females with ages ranging between $25-55$ years. All participants were scanned with panoramic and cone beam computed tomography (CBCT).
\end{abstract}

Results: ROC curve analysis of panoramic views showed diagnostic accuracy $76.4 \%$ while diagnostic accuracy of CBCT (TMJ views) was $81.3 \%$ and CBCT (Sagittal views) showed $82.6 \%$ diagnostic accuracy.

Conclusion: Panoramic images can be used as a preliminary valuable tool for evaluation of osteoarthritic osseous changes equivalent to $\mathrm{CBCT}$ images as it revealed relatively high sensitivity compared to CBCT images.

KEYWORDS: Diagnostic imaging, CBCT, TMJ osteoarthritis.

\section{INTRODUCTION}

Osteoarthritis (OA) is a chronic, complex and progressive disease which is characterized by pain, stiffness and loss of function of the affected joint. Temporomandibular joint osteoarthritis (TMJ-OA) is one of most well-known disorders affecting the temporomandibular joint (TMJ), it is more frequent in females and its prevalence increases in relation to age. Many factors such as bruxism, uni-lateral chewing, hereditary factors and internal derangement are considered responsible for the development of TMJ-OA. The diagnostic criteria for TMJ-OA are history of TMJ noise during function or movement upon examination or within the last 30 days together with crepitus at movement during palpation. Histologic and morphologic

\footnotetext{
* Associate Professor of Oral and Maxillofacial Radiology, Faculty of Dentistry, Cairo University, Egypt.
} 
changes to the joint tissue begin, resulting in typical morphological changes to the TMJ condyle including flattening, sclerosis, osteophyte formation, erosion, and subcortical bone cysts. These clinical criteria together with histologic and morphologic changes should be adjunct with imaging to complete the diagnosis of TMJ-OA. ${ }^{(1-8)}$

The small nature of TMJ components makes its imaging inherently difficult, as well as the indistinct image of TMJ results from superimpositions with the cranial base. Imaging findings have a great impact on the clinician's treatment of patients with TMD. Many imaging modalities have been used to evaluate the TMJ. Panoramic radiography, conventional linear or complex motion tomography and computed tomography (CT) are used to assess the osseous components of the joints. ${ }^{(9,10)}$

Nowadays, with the presence of our great tool and state of art in dentistry, CBCT has overcome all the intrinsic weaknesses of conventional imaging, providing clear non distorted image in all planes by the use of magic manipulative tool which is rotation that enables us to rotate either the image itself or the structure of interest in any direction to get the maximum information and the best outcomes. ${ }^{(7,11-14)}$

\section{PATIENTS AND METHODS}

25 patients were enrolled in the current investigation, divided into two groups, group (1) the diseased group including fifteen patients (30 joints) were evaluated with the following eligibility criteria:

1- Diagnosed as having osteoarthritis

2- Newcomers to the university hospital of Rheumatology and Rehabilitation Department, Faculty of Medicine, Cairo University.

3- Showed signs and symptoms of the disease but did not have medical help ever.

Group (2) the control group including: Ten normal asymptomatic healthy patients (20 joints) they were seeking for treatment of other dental problems, in the two groups enrolled in this study all participants were females with ages ranging between 25-55 years.

\section{Inclusion and Exclusion Criteria}

\section{Inclusion Criteria}

\section{I-Diseased group (group 1)}

Patients with clinical signs and symptoms denoting osteoarthritic changes of the TMJ such as headache, pain, tenderness, joint sounds and irregular or deviating jaw function were included as one of the diseased group members.

\section{II-Control group (group 2)}

Participants who proved to be normal, healthy and free from any TMJ troubles based on clinical examination (free from TMJ complaints and without history of any traumatic injuries or orthodontic treatment) were also included in the study as one of the control members but they were seeking for treatment of other dental problems.

\section{Exclusion Criteria for Group One Patients}

Subjects with any inflammatory rheumatic diseases or any other systemic diseases affecting the skeletal system were excluded. For standardization of measurements of maximum mouth openings, patients with missing anterior teeth were excluded.

All of the included patients were subjected to the following methods of evaluation: History taking of osteoarthritic TMJ complains, the clinical characteristics of the TMJ-related problems in every subject were recorded through intra and extra-oral examination of the TMJ and radiographic examination.

\section{1- Clinical examination}

The clinical characteristics of the TMJ-related problems in every subject were recorded through intra and extra-oral examination of the TMJ in order to elicit all the following signs and symptoms using inspection and palpation: (Lin et al 2007) ${ }^{(15)}$ 


\section{1- Palpation for tenderness of TMJ}

Bidigital lateral palpation on both sides of the face was performed (using index and middle finger) with firm pressure on the lateral pole of the condyle (just in front). Posterior palpation of the TMJ was done by gently placing the little finger into the outer portion of the external auditory canal. Positive results were considered if the patient felt pain on pressure.

2- Difficulty in opening and closing the mouth or trismus.

3- Auscultation to detect the click and crepitus sound of TMJ during mandibular opening and closing movements.

4- Deviation of the mandible when attempting to open the mouth.

\section{2- Radiographic examination}

**All the subjects were submitted to two imaging protocols as follows:

\section{A-Panoramic Imaging}

Panoramic images were performed at the Oral Radiology Department, Faculty of Dentistry, Cairo University: Using multimodal panoramic machine (Planmeca OY Asentajankatu 6, 00880 Helsinki, Finland). The parameters used were 7072 killovoltage $(\mathrm{kVp})$ according to the patient's age and sex, 13 milliampere $(\mathrm{mA})$ and 13 seconds exposure time.

\section{B-Cone beam Computed Tomographic Imaging (CBCT)}

\section{Technical Concideration}

CBCT images were taken using (i-CAT) Image Sciences International Hatfield, Pa USA, The sagittal and coronal images were obtained using dental mode at (120) kV, (5) mA, (10) seconds.

\section{Image Analysis}

Diagnostic accuracy for the detection of flattening, defects, resorption and osteophytes in the various osseous joint components (condylar head, articular eminence and articular fossa) on all lateral and frontal imaging modalities were assessed.

The analysis was performed at two different sessions with a week interval in between the sessions. The differences between the observer and himself were expressed as the sensitivity and specificity of each modality after applying statistical analysis of observer' results (intra-observer variability).

The consensus naked-eye inspection for the radiographic interpretation of the images of the healthy volunteers served as the gold stand for the radiographic examinations.

The consistent radiographic finding was considered the gold standard for performing the association statistical analysis tests.

The images were assessed as follows:

\section{Osseous changes of the condyles ( $\mathrm{Su} N$ et al $2014^{(7)}$ and Alexiou et al 2009(16))}

The following radiographic views were used to assess the osseous changes of the condyles:

1- Conventional panoramic images. (Figure.1)

2- CBCT images (sagittal, coronal and axial cuts as well as TMJ views). (Figure. 2)

These osseous changes were defined as follows:

1. Flattening: defined as a flat bony contour deviating from the convex form; loss of convexity of the bony outline.

2. Erosion: defined as an area of decreased density of the cortical bone and the adjacent subcortical bone.

3. Osteophyte: defined as marginal local area of bony outgrowths on the condyle arising from the exterior surface. 
4. Sclerosis: defined as an area of increased density of cortical bone extending into the bone marrow.

5. Resorption: defined as partial loss of condylar head.

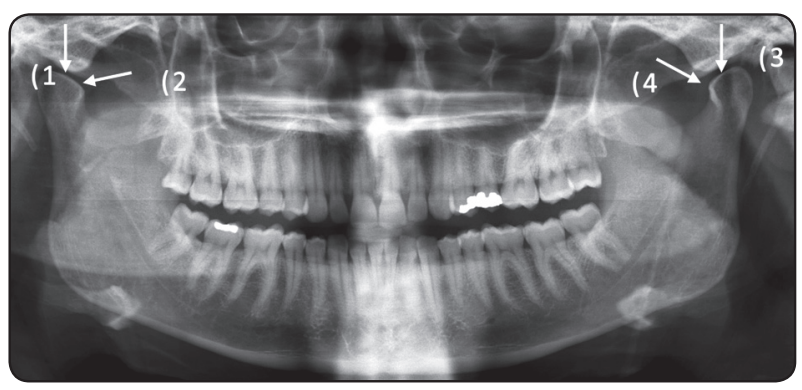

Fig. (1) The panoramic radiograph of the patient reveals slight flattening of the right condyle (1). with clearly demonstrated osteophyte on the medial aspect of the right condyle (2). There is also slight flattening (3) with clearly demonstrated osteophyte on the medial aspect of the left condyle (4)

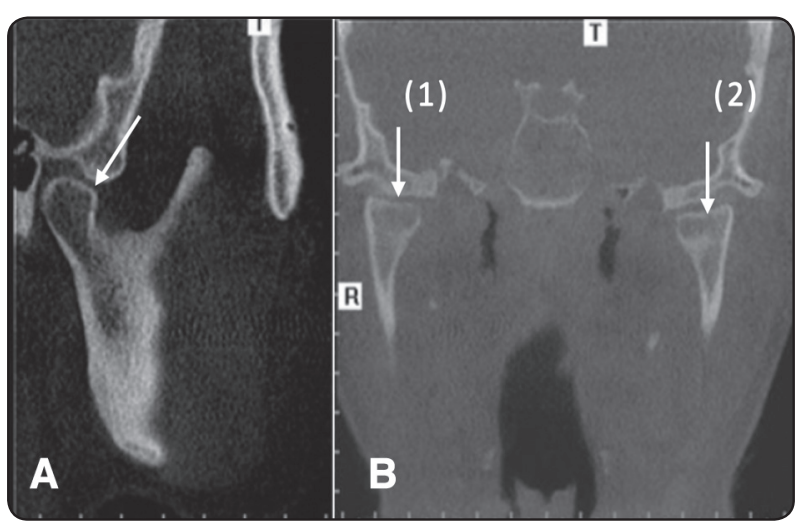

Fig. (2) A) Sagittal images (closed) perfectly depicting osteophyte formation on the right condyle.B) Coronal view of this patient can clearly delineate the flattening of right (1) and left condyles (2)

\section{Statistical Analysis:}

Receiver operating characteristics (ROC) curve between sensitivity and specificity is a useful method to evaluate the performance of a diagnostic test in classification of subjects into two categories (say) positive and negative. ROC curve may be used to judge how well the test performs. If area under the curve (AUC) is near 1 it has higher chance of correct classification and when it is near 0 , higher chance of incorrectly classifying in opposite group. If the $95 \%$ confidence interval of the AUC does not include the 0.5 value, then there is evidence that the test does have an ability to distinguish between the two groups. A high sensitivity results in low number of false negative cases while high specificity leads to low number of false positive cases. Sensitivity, specificity and diagnostic accuracy were calculated and compared between the different modalities using repeated measures ANOVA.

Inter-observer agreement ranged from $(0.380-$ $1.000)$ denoting fair to perfect reliability.

\section{RESULTS}

\section{ROC Curve Analysis}

\section{Diagnostic Accuracy of Panoramic Images}

ROC curve analysis of panoramic views showed a sensitivity of $78.1 \%$, specificity of $72.9 \%$ and the diagnostic accuracy was $76.4 \%$

\section{Diagnostic Accuracy of CBCT(TMJ) Views}

ROC curve analysis of CBCT (TMJ views) showed a sensitivity of $85.4 \%$, specificity of $72.9 \%$ and the diagnostic accuracy was $81.3 \%$

\section{Diagnostic Accuracy of CBCT(Sagittal) Views}

ROC curve analysis of CBCT (Sagittal views) showed a sensitivity of $85.4 \%$, specificity of $77.1 \%$ and the diagnostic accuracy was $82.6 \%$

\section{Diagnostic Accuracy of CBCT(Coronal) Views}

ROC curve analysis of CBCT (Coronal views) showed a sensitivity of $56.3 \%$, specificity of $100 \%$ and the diagnostic accuracy was $70.8 \%$

\section{Diagnostic Accuracy of CBCT(Axial) Views}

ROC curve analysis of CBCT (Axial views) showed a sensitivity of $32.3 \%$, specificity of $91.7 \%$ and the diagnostic accuracy was $52.1 \%$ 


\section{Comparison between areas under the ROC curve}

Regarding detection of morphologic changes, there was no statistically significant difference between (CBCT TMJ views=0.792), (CBCT Sagittal views $=0.813),($ CBCT Coronal views $=0.781)$ and (panorama $=0.755)$; all showed the statistically significantly highest mean areas under the curve (denoting the highest accuracy).(CBCT Axial views $=0.620$ ) showed the statistically significantly lowest mean area under the curve (denoting the lowest accuracy) and $\mathrm{p}$ value is $<0.001$.

\section{DISCUSSION}

The location of the bone of the mandibular condyles just beneath the fibrocartilage covers the TMJ and the sophisticated movement system of the TMJ, making it particularly vulnerable to inflammatory damage and offering a valuable model for studying arthritic bony changes. ${ }^{(6,17)}$

a variety of symptoms may be found in OA patients including; jaw and muscle pain, a grating sensation, called crepitus, pain when opening and closing the mouth, referred pain from the jaw to the neck, head and arms, stiffness of the neck, earaches, headaches, misalignment of the upper and lower teeth. ${ }^{(18,19)}$

That's why patients with clinical signs and symptoms denoting osteoarthritic changes of the TMJ such as headache, pain, tenderness, joint sounds and irregular or deviating jaw function were included as one of the diseased group members in the present study.

Once and for all panoramic radiography is helpful in evaluating gross TMJ osseous pathology. Despite this technique is simple and relatively inexpensive, it suffers from many weaknesses makes it worthless for TMJ assessment. First, limited view of the entire articular surface of the TMJ. Second, poor reliability and low sensitivity for detecting TMJ osseous changes narrowing the diagnostic value in radiographic evaluation of the TMJ. This creates obstacles in the detailed assessment of changes in surface morphology of the condyle and the fossa. ${ }^{(9,20)}$

Cone-beam computed tomography (CBCT) provides practitioners with exquisite manipulative tools beyond the ordinary softwares capabilities, Thus, enabling them to sail into the three dimensional journey and back with all the needed information to get the best long lasting prognosis. ${ }^{(10)}$

In this study ROC curve analysis of panoramic views showed diagnostic accuracy $76.4 \%$ while diagnostic accuracy of CBCT (TMJ views) was $81.3 \%$ and CBCT (Sagittal views) showed $82.6 \%$ diagnostic accuracy

Regarding assessment of joint morphology, there was no statistically significant difference between (CBCT TMJ view), (CBCT sagittal view) and (CBCT coronal view); all showed the statistically significantly highest mean areas under the curve (denoting the highest accuracy).CBCT axial view showed the statistically significantly lowest mean area under the curve (denoting the lowest accuracy).

In the current study, it was found that condylar lesions were detected more frequently than temporal bone lesions which are in agreement with Honda et al 2006 ${ }^{(21)}$ observations who confined their examination to the mandibular condyle. However, on the contrary Alexiou et al $\mathbf{2 0 0 9 ^ { ( 1 6 ) }}$ results found that the osseous changes of the mandibular fossa were common in patients with osteoarthritis.

Honey et al 2007 ${ }^{(22)}$ illustrated that osteoarthritis of the mandibular condyle not only produces flattening of the surface, as simulated by the defect, but might also be associated with morphologic and surface changes such as sclerosis, osteophyte formation, and reduced interarticular space. These findings come in agreement with our results and with the results of Alexiou et al 2009 ${ }^{(16)}$ who assessed the most frequent radiographic findings in patients with TMJ degenerative arthritis. 
In agreement with current results Honey et al $\mathbf{2 0 0 7}^{(22)}$ found that the CBCT images provided significantly greater accuracy than TMJ-specific panoramic projections in the detection of simulated condylar defects. This result was expected because CBCT display of TMJ volumetric data provides multiple corrected sagittal as thin as $0.3 \mathrm{~mm}$ slices of high contrast with no structural superimposition or tomographic blur, as do the other images. Interestingly, Honey et al $\mathbf{2 0 0 7}^{(\mathbf{2 2})}$ did not find improved accuracy for detecting TMJ defects using conventional panoramic radiography. These results are consistent with ours. This finding might be related to the inherent superimposition of structures, variable distortion, and the focal trough limitations of this modality.

Against our results, Hintze el al 2007 and $\mathbf{2 0 0 9}^{(23,24)}$ showed high specificity values for all changes with panoramic method. That may be explained by the fact that all methods under evaluation were effective for the identification of joint components without any pathological changes. However, the high specificities could also indicate method limitations. If the radiographic methods under evaluation are unable to depict truly present TMJ bone changes then the observers would not be able to perceive them, and therefore they recorded the joint component as having no change - the same as being sound.

\section{CONCLUSION}

Anoramic images can be used as a preliminary valuable tool for evaluation of osteoarthritic osseous changes equivalent to $\mathrm{CBCT}$ images as it revealed relatively high sensitivity compared to $\mathrm{CBCT}$ images.

\section{REFERENCES}

1- Nitzan DW, Benoliel R, Heir G, Dolwick F: Pain and dysfunction of the temporomandibular joint. In: Sharav Y, Benoliel R (Eds). Orofacial pain and headache. London: Elsevier Health Sciences 149, 2008.
2- Tanaka E, Detamore MS, Mercuri LG: Degenerative disorders of the temporomandibular joint: etiology, diagnosis, and treatment. J Dent Res 2008;87: 296-307.

3- Barros Vde M, Seraidarian PI, Cortes MI, de Paula LV: The impact of orofacial pain onthe quality of life of patients with temporomandibulardisorder. J Orofac Pain.2009; 23: 28-37.

4- CevidanesL. H. S., Hajati A.-K, Paniagua B, LimP. F., Walker D. G, G. Palconet, Nackley A. G, Styner M, Ludlow J. B and Zhu Hand Phillips C: Quantification of condylar resorption in temporomandibular joint osteoarthritis. Oral Surg Oral Med Oral Pathol Oral Radiol Endod 2010;110:110-117.

5- Liu F, Steinkeler A. Epidemiology, diagnosis, and treatment of temporomandibular disorders. Dent Clin North Am 2013;57: 465-79.

6- LimM-J, Lee J-Y: Computed tomographic study of the patterns of oesteoarthritic change which occur on the mandibular condyle. Journal of Cranio-Maxillo-Facial Surgery.2014; 1-6.

7- Su N, Liu Y, Yang X, Luo Z, Shi Z: Correlation between bony changes measured with cone beamcomputed tomography and clinical dysfunction index in patients withtemporomandibular joint osteoarthritis:Journal of CranioMaxillo-Facial Surgery 2014; 1-6.

8- Comert Kilic S, Kilic N, Sumbullu MA: Temporomandibular joint osteoarthritis: cone beam computed tomography findings, clinical features, and correlations. Int J Oral MaxillofacSurg 44:1268, 2015.

9- Barghan S, Tetradis S and Mallya SM: Application of cone beam computed tomography for assessment of the temporomandibular joints. Australian Dental Journal 2012; 57: (1 Suppl): 109-118.

10- Patel A, Ching Tee B, Fields H, Jones E, Chaudhry J and Sun Z: Evaluation of cone-beam computed tomography in the diagnosis of simulated small osseous defects in the mandibular condyle. Am J OrthodDentofacialOrthop 2014; 145:143-56.

11- Paniagua B, Cevidanes L, Walker D, Zhu H, Guo R and Styner M: Clinical application of SPHARM-PDM to quantify temporomandibularjoint osteoarthritis. Computerized Medical Imaging and Graphics 2011; 35: 345-352.

12- Akiyama K, Mizui T, Baba S, Takahashi T and Ishimaru J: Imaging the temporomandibular joint by double contrast 
cone-beam computedarthrotomography.Journal of Oral and Maxillofacial Surgery, Medicine, and Pathology 2012; 24: $23-26$.

13- Kurt H,Orhan K, Aksoy S, Kursun S, Akbulut N, and BilecenogluB: Evaluation of the superior semicircular canal morphology using cone beam computed tomography: a possible correlation for temporomandibular joint symptoms. Oral Surg Oral Med Oral Pathol Oral Radiol 2014; 117: e280-e288.

14- Larheim, T. A., Hol, C., Ottersen, M. K., Mork-Knutsen, B. B., \& Arvidsson, L. Z. The Role of Imaging in the Diagnosis of Temporomandibular Joint Pathology. Oral and maxillofacial surgery clinics of North America. 2018.

15- Lin Y, Hsu M, Yang J, Liang T, Chou S and Lin H: Temporomandibular joint disorders in patient with rheumatoid arthritis. J Chin Med Assoc 2007; 70 (12): 527- 534.

16- Alexiou KE, Stamatakis HC and Tsiklakis K: Evaluation of the severity of temporomandibular joint osteoarthritic changes related to age using cone beam computed tomography. Dentomaxillofacial Radiology 2009; 38: 141-147.

17- CevidanesL.H.S, Walker D, Schilling J, Sugai J, Giannobile W, Paniagua B, et al: 3D osteoarthritic changes in TMJ condylar morphology correlate with specific systemic and local biomarkers of disease. Osteoarthritis and Cartilage 2014; 22 : 1657-1667.

18- Maydana AV, Tesch RS, Denardin OVP, Ursi WJS and Dworkin SF: Possible etiological factors in temporomandibular disorders of articular origin with implications for diagnosis and treatment. Dental Press Journal of Orthod 2010 May-June; 15(3): 78-86.

19- Hawkins R: Jaw Osteoartheritis Symptoms. Health Articles online; osteoarthritis , 2011.

20- Ikedaa K and Kawamura A: Assessment of optimal condylar position with limited cone-beam computed tomography. Am J OrthodDentofacialOrthop 2009; 135: 495-501.

21- Honda K, Larheim TA and Maruhashi K: Osseous abnormalities of the mandibular condyle: diagnostic reliability of cone beam computed tomography compared with helical computed tomography based on an autopsy material. DentomaxillofacRadiol 2006; 35:152-57.

22- Honey OB, Scarfe CW, Hilgers MJ, Klueber K, Silveira AM, Haskell BS and Farmang AG: Accuracy of conebeam computed tomography imaging of the temporomandibular joint: Comparisons with panoramic radiology and linear tomography.American Journal of Orthodontics and Dentofacial Orthopedics 2007; 132(4): 429-438.

23- Hintze H, Wiese M and Wenzel A: Comparison of three radiographic methods for detection of morphological temporomandibular joint changes: panoramic, scanographic and tomographic examination. Dentomaxillofacial Radiology 2009; 38: 134-140.

24- Hintze H, Wiese $M$ and Wenzel A: Cone beam CT and conventional tomography for the detection of morphological temporomandibular joint changes. Dentomaxillofacial Radiology2007; 36: 192-7. 